\title{
Design of multi-component periodic maintenance programs with single-component models
}

\author{
Joachim Arts ${ }^{1}$ and Rob Basten ${ }^{2}$ \\ ${ }^{1}$ University of Luxembourg, Luxembourg Centre for Logistics and Supply Chain \\ Management, Faculty of Law, Economics and Finance, joachim.arts@uni.lu \\ ${ }^{2}$ Eindhoven University of Technology, School of Industrial Engineering, r.j.i.basten@tue.nl
}

January 30, 2018

\begin{abstract}
Capital assets, such as wind turbines and ships, require maintenance throughout their long life times. Assets usually need to go down to perform maintenance and such downs can be either scheduled or unscheduled. Since different components in an asset have different maintenance policies, it is key to have a maintenance program in place that coordinates the maintenance policies of all components, so as to minimize costs associated with maintenance and downtime. Single component maintenance policies have been developed for decades, but such policies do not usually allow coordination between different components within an asset. We study a periodic maintenance policy and a condition based maintenance policy in which the scheduled downs can be coordinated between components. In both policies, we assume that at unscheduled downs, a minimal repair is performed to keep unscheduled downtime as short as possible. Both policies can be evaluated exactly using renewal theory, and we show how these policies can be used as building blocks to design and optimize maintenance programs for multi-component assets.
\end{abstract}

Keywords: multi-component systems, condition based maintenance, group maintenance, renewal theory, delay time model

\section{Introduction}

Capital assets, such as ships, production lines, wind turbines, or weapon systems require maintenance throughout their long life times. Capital assets are crucial to the primary processes of their users, so unexpected failures that lead to unscheduled downtime of an asset have a large negative impact. Therefore, asset owners prefer to perform preventive maintenance to avoid unscheduled downs. This has many 
advantages over corrective maintenance: Maintenance may be performed at moments that the capital asset is not being used anyway or when it can be shut down without incurring too high downtime costs. Furthermore, collateral damage on other components can be avoided and required resources, such as spare parts, service engineers and tooling, can be available at the right place at the right time. Finally, maintenance on multiple components can be clustered; we come back to this below.

Historically, periodic maintenance has been prevalent. Maintenance is then triggered by a usage related measure such as calendar time in service, number of sheets printed, or running time. The first models have been proposed over fifty years ago by Barlow and Hunter (1960). Condition based maintenance is becoming more popular because the condition of components becomes easier and cheaper to measure with modern sensor technology. It can be measured, for example, by the concentration of ferrous particles in lubrication fluid, the vibration amplitude, or the temperature at moments of peak load. A sensor may be installed that measures continuously or the condition may be inspected with a certain interval. Condition monitoring may not be possible (cost effectively) for all components in a capital asset. Therefore, a periodic or corrective maintenance policy is used for some components, while a condition based maintenance policy is used for other components.

Since a system usually needs to be down in order to replace or maintain any of its components, while the consequences of downtime are typically severe, maintenance programs are usually designed for systems as a whole. Downtime, especially unscheduled downtime, and maintenance costs then need to be balanced. Therefore, maintenance programs often have scheduled downs (also called scheduled downtimes) during which it is possible to do maintenance, inspections, and/or replacements for any component in the system. At these moments, we need to perform maintenance in such a way that the risk of an unscheduled down due to failure before the next scheduled down is minimized. We are aware of one work in which a maintenance program is optimized for a multi-component system in which different components may require different maintenance policies: Zhu (2015). Our approach is in line with his, in that we propose two single component models, one for periodic preventive maintenance and one for condition based maintenance, that can be used in a larger framework to optimize a maintenance program for a complete system. We discuss the work of Zhu (2015) in more detail in Section 2.

The periodic maintenance model that we propose is a generalization of the block replacement policy with minimal repair upon failure (Policy II of Barlow and Hunter, 1960). In the latter policy, there is a schedule with a certain interval $\tau$ at which a component is replaced. If the component fails during an interval, then a minimal repair is performed, meaning that the component's state is restored to a state that is statistically identical to the state just prior to the component's failure. In other words, the failure rate immediately after the minimal repair is equal to that just before the failure. Our policy has an additional control parameter $n$, that differs per component: A component is replaced after every $n \tau$ time units if nothing happens, i.e., at every $n$-th scheduled down. If a failure occurs at some point, then a minimal repair is performed immediately, and at the next scheduled down, a replacement is 
performed. Notice that throughout the paper, at scheduled downs, instead of replacements also a repair or preventive maintenance action may be performed that brings the state of the component back to as-good-as-new. From a modeling point of view, this is equivalent.

The condition-based model that we propose uses the delay time model to model degradation (DTM; introduced by Christer, 1982; Christer and Waller, 1984). This is a semi-Markov model in which a component can be in three different states: good, defect and failed. The time a component remains in the good state after replacement, known as the time-to-defect, is assumed to be exponential. This assumption is natural in several common settings. For example, when manual inspections are performed on many components, it is often not possible to measure and store the exact degradation level. Therefore, it is only checked if the degradation has passed a certain threshold. This means that although the degradation may follow a certain process, from a planning point of view, the time to defect is random. When the component becomes defect, there is a generally distributed time to failure, also known as the delay time. We assume that defects are not self-announcing, while failures are. Therefore, we require inspections to find defects. These are performed at every $n$-th scheduled down, i.e., after every $n \tau$ time units. If the component turns out to be defect, it is replaced. If a component fails in between two inspection moments, then a minimal repair is performed, and the component is replaced at the next scheduled down.

The key contributions of our paper are as follows. We propose two single-item maintenance models with minimal repair, which means that (1) these models are useful in practice in situations where performing a complete repair or replacement is too time-consuming to perform outside of a scheduled down and (2) we can evaluate the models exactly, which is not possible for the models that Zhu (2015) proposes. Further, (3) by design, these models are fit for use in the optimization of a maintenance program for a multi-component asset.

The remainder of this paper is structured as follows. In Section 2, we review the related literature, focusing on multi-component maintenance optimization. Our key contribution, the periodic and condition based models that we propose, are in Sections 3 and 4, respectively. Next, in Section 5, we give examples of how our models can be used and how they can be combined to optimize a multi-component maintenance program. We conclude in Section 6.

\section{Literature}

There is much literature on single-component maintenance optimization, starting with the work on optimizing the periodic maintenance interval by Barlow and Hunter (1960, Policy I). We will not treat these models here; we refer to any textbook on reliability engineering or maintenance optimization (e.g., Jardine and Tsang, 2006; Pintelon and Van Puyvelde, 2006). However, the block replacement policy is a relevant single-component maintenance model, as explained in Section 1: If there are many identical 
components, then it makes sense to preventively replace all of them at the same time. Therefore, the block policy is also a single-item maintenance policy that can be used for multi-item maintenance optimization. The block replacement policy with minimal repairs upon failure has been introduced by Barlow and Hunter (1960, Policy II) and with complete repairs or, equivalently, replacements by Barlow and Proschan (1965, pp.95-96). The (analysis of) the block policy has been extended to allow not preventively replacing just after a corrective replacement (Berg and Epstein, 1976; Bajestani and Banjevic, 2016), opportunistic inspections for latent failures between periodic replacement times (Taghipour and Banjevic, 2012), imperfect repairs (which is between replacement and minimal repair) (de Toledo et al., 2016), and unpunctual implementation of periodic replacement times (He et al., 2017).

The condition based maintenance model that we propose builds on the delay time degradation model as proposed by Christer (1982). Recent contributions in delay time models include Driessen et al. (2017) who allow for imperfect inspections whose probability of misreading change over time, Jiang (2017) who allow for postponing the time until the first inspection, and Yang et al. (2017) who extend the degradation model to allow for sudden shock failure. None of these models ensure that the inspection schedule remains unchanged after an unexpected event such as a failure, whereas the model we propose in this paper does. This allows us to coordinate inspections over multiple components so as to leverage economic dependence.

Almost all real-life systems consist of multiple different components, so that it is not only relevant to decide on which maintenance policy to use for each component (e.g., corrective maintenance, periodic maintenance, or condition-based maintenance) and to optimize the policy per component, but also to cluster the maintenance of different components. The reason to cluster maintenance on multiple components is the dependencies between components. Often, three types of dependencies are considered (see, e.g., Thomas, 1986; Nicolai and Dekker, 2008; Van Horenbeek and Pintelon, 2013): economic, structural and stochastic dependencies. Economic dependence is virtually always present: It means that the costs of performing two maintenance actions at the same time is not the same as the summation of the costs of performing each of the two maintenance actions. Most common is a positive economic dependency when there are set up costs that are shared. For example, a service engineer needs to go to the capital asset only once to perform two maintenance actions. Structural dependence means that the structure of the asset is such that to maintain one component, another may need to be dismantled (see, e.g. Levi et al., 2014). Finally, stochastic dependence means that the degradation processes of different components are correlated. This usually happens when the degradation level of one component accelerates the degradation of another component (see e.g. Liu et al. (2016) or Bian and Gebraeel (2014)).

All three types of dependencies have been discussed in the literature on multi-component maintenance optimization. This literature has been reviewed several times; see, for instance, Thomas (1986) and Nicolai and Dekker (2008). Both reviews are structured according to the dependencies. Interestingly, the former review does not mention condition-based maintenance at all, while the latter review 
mentions only one paper in which condition-based maintenance is considered.

We further focus on economic dependence, since that is the type of dependence that we also consider. We then notice that virtually all of this literature considers multiple components that all use the same maintenance policy. Many authors have considered clustering of maintenance on components that each use a periodic maintenance policy. Older examples are Wildeman et al. (1997) and Van Dijkhuizen and Van Harten (1997), while a newer examples include Levi et al. (2014), Zhang and Yang (2015) and Barron (2015). Only few articles consider clustering of maintenance on components that each use a conditionbased maintenance policy. Examples are Castanier et al. (2005), Tian and Liao (2011), De Jonge et al. (2016) and Olde Keizer et al. (2016). Olde Keizer et al. (2017) review this literature.

To the best of our knowledge, the only work in which the maintenance program is optimized for a complete system that consists of components with different maintenance policies, is the PhD thesis of Zhu (2015). He has proposed a framework that functions as follows. Maintenance moments, called scheduled downs, are scheduled for the complete capital asset at constant intervals of length $\tau$. In addition, unscheduled downs may occur due to failures in components. At both scheduled and unscheduled downs, maintenance can be performed at each of the components for which a periodic or condition based maintenance policy is selected (the single-item models per component have also been published as Zhu et al., 2016, 2017, respectively). The long term average costs per time unit can be evaluated for each of the components for a given $\tau$. Summing those costs for all components and adding joint setup costs (translated to costs per time unit) leads to the total costs per time unit for the complete asset. $\tau$ is then increased in small steps between a lower and an upper bound to find the approximately optimal scheduled downs interval $\tau$. It is approximately optimal for two reasons: First, $\tau$ is varied in small steps, while the true optimum may be in between two steps. Second, the cost evaluation for each component using a periodic or condition based maintenance policy is approximate.

Our approach is similar to that of Zhu (2015). However, there are two key differences. First, the single-component models that we propose can be evaluated exactly, often in closed form, thus getting rid of the second type of approximation that Zhu (2015) makes. Since the step size, and thus the first approximation, can be arbitrarily small, this allows for an arbitrarily close approximation of the true optimal cost rate for a complete asset. Second, we use scheduled downs as opportunities to perform maintenance on all components, but we do not use unscheduled downs as such opportunities. In the settings that we have in mind, ship maintenance, for instance, performing a minimal repair and having the system running again, is of key importance and often the only thing that is possible since resources required for full replacement or maintenance are not available except during scheduled downtimes. 


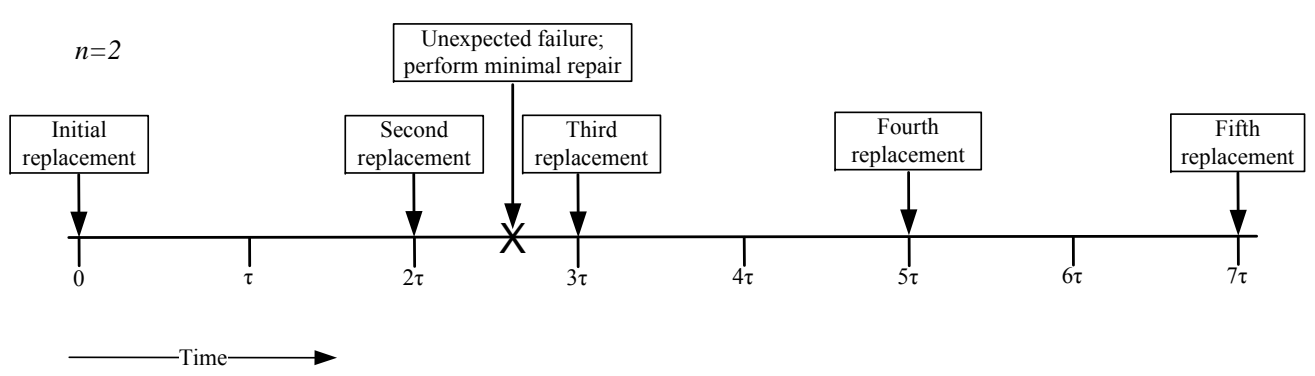

Figure 1: Sample path of the PUBM policy with $n=2$.

\section{Periodic usage based maintenance model}

In this section, we present the first single-item maintenance model we propose. We consider a component with a random lifetime, $T$, with distribution $\mathbb{P}(T \leq x)=F_{T}(x)$, density $f_{T}(x)=\frac{d F_{T}(x)}{d x}$, and failure rate (or hazard rate) $h_{T}(x)=f_{T}(x) /\left(1-F_{T}(x)\right)$. Every $\tau$ time units, there is a scheduled down during which this component can undergo planned replacement to restore the component to as-good-as-new condition. We propose the following policy: After the preventive replacement of a component, we replace it preventively again at the $n$-th scheduled down, $n \in \mathbb{N}$. If the component fails before the $n$-th scheduled down, we apply a minimal repair and replace at the next scheduled down. Such a minimal repair will bring the condition of a component back to a condition that is statistically identical to the state just prior to its failure. That is, after a minimal repair, the remaining lifetime distribution of component is identical to the remaining lifetime distribution conditional on no failure having occurred yet. We call this the periodic usage based maintenance (PUBM) policy. A schematic representation of a sample path under the PUBM policy is given in Figure 1. A planned preventive replacement at a scheduled down costs $C_{p} \geq 0$, while a replacement after a failure has occurred costs $C_{u} \geq 0$. Each minimal repair costs $C_{r} \geq 0$.

We note that the PUBM policy may not perform better than classical policies such as the age replacement policy in all settings. However, due to its periodic nature, it can leverage economic dependencies between different components in a system, whereas the age replacement policy cannot.

Note that both $n$ and $\tau$ are policy parameters subject to optimization. (In a multi-component system, $\tau$ will be common among different components, but $n$ can be unique to each component.) Observe further that if $n=1$, the PUBM policy reduces to a block policy with minimal repair. Similarly, if $\tau \rightarrow 0$ and $n \rightarrow \infty$ such that $n \tau \rightarrow t$, then the PUBM policy reduces to a regular age-based policy with replacement age $t$. Thus, the PUBM policy also applies to settings where the classical age-based policy and block replacement policy with minimal repair apply. 


\subsection{Performance evaluation}

Observe that the cost incurred by a PUBM policy can be described by a renewal reward process. The renewal points are the moments where a component is replaced at a scheduled downtime. The expected renewal cycle length, ECL, for a PUBM policy is found by conditioning on the interval in which a component fails for the first time:

$$
\begin{aligned}
E C L & =\sum_{k=1}^{n-1} k \tau \mathbb{P}((k-1) \tau \leq T \leq k \tau)+n \tau \mathbb{P}(T \geq(n-1) \tau) \\
& =\sum_{k=1}^{n-1} k \tau\left[F_{T}(k \tau)-F_{T}((k-1) \tau)\right]+n \tau\left(1-F_{T}((n-1) \tau)\right) .
\end{aligned}
$$

It is more involved to determine the cost during a renewal cycle. The crux is finding the expected number of minimal repairs that will be conducted during a renewal cycle. For the exposition, it is useful to introduce the random variable $Y\left(t_{1}, t_{2}\right)$ that denotes the number of minimal repairs in the interval $\left(t_{1}, t_{2}\right)$ that belong to a renewal cycle that started at time $0\left(t_{1}<t_{2}<n \tau\right)$. (Note that minimal repairs in the interval $\left(t_{1}, t_{2}\right)$ that occur after a renewal after time 0 but before time $t_{1}$ do not belong in the first renewal cycle.) The expected number of minimal repairs in the interval $(0, \tau)$ is given by $\mathbb{E}[Y(0, \tau)]$ under this new definition. Observe that (cf. Barlow and Hunter, 1960) $\mathbb{E}[Y(0, \tau)]=\int_{x=0}^{\tau} h_{T}(x) d x$ because $Y(0, \tau)$ corresponds with the number of minimal repairs in a block replacement policy with block length $\tau$. The expression for $\mathbb{E}[Y((k-1) \tau, k \tau)]$ with $k \geq 1$ is slightly more complicated and given in Proposition 1.

Proposition 1. The expected number of minimal repairs under a PUBM policy in the interval $((k-1) \tau, k \tau)$ for $k \in\{1, \ldots, n\}$ that belong to a renewal cycle started at time 0 satisfies:

$$
\mathbb{E}[Y((k-1) \tau, k \tau)]=\left(1-F_{T}((k-1) \tau)\right) \int_{x=(k-1) \tau}^{k \tau} h_{T}(x) d x
$$

Proof. We condition on whether the renewal cycle that starts at time 0 is still ongoing in the interval $((k-1) \tau, k \tau):$

$$
\begin{aligned}
\mathbb{E}[Y((k-1) \tau, k \tau)]= & \mathbb{E}[Y((k-1) \tau, k \tau) \mid T>(k-1) \tau] \mathbb{P}(T>(k-1) \tau) \\
& +\mathbb{E}[Y((k-1) \tau, k \tau) \mid T \leq(k-1) \tau] \mathbb{P}(T \leq(k-1) \tau) \\
= & \mathbb{E}[Y((k-1) \tau, k \tau) \mid T>(k-1) \tau]\left(1-F_{T}((k-1) \tau)\right) \\
= & \left(1-F_{T}((k-1) \tau)\right) \int_{x=(k-1) \tau}^{k \tau} h_{T}(x) d x
\end{aligned}
$$

The first equality uses the law of total probability, the second follows because $\mathbb{E}[Y((k-1) \tau, k \tau) \mid T \leq$ $(k-1) \tau]=0$ by definition of the PUBM policy. 
Now it is straightforward to verify that the expected costs during a renewal cycle, ECC, of the PUBM policy satisfy:

$$
E C C=C_{p} \mathbb{P}(T \geq n \tau)+C_{u} \mathbb{P}(T<n \tau)+C_{r} \sum_{k=1}^{n} \mathbb{E}[Y((k-1) \tau, k \tau)] .
$$

Now by renewal reward theory (e.g. Theorem 2.2.1 of Tijms, 2003), the average cost rate of a PUBM policy with parameters $n$ and $\tau$ is given by $C^{P U B M}(n, \tau)=E C C / E C L$ where $E C L$ is given by equation (1) and ECC is given by equation (4) with $\mathbb{E}[Y((k-1) \tau, k \tau)]$ as given in Proposition 1.

Remark 1. The computation of $\mathbb{E}[Y((k-1) \tau, k \tau)]$ can be done in closed form if $T$ has a Weibul distribution. In particular, it is straightforward to show that if $F_{T}(x)=1-\exp \left(-(x / \alpha)^{\beta}\right)$, with $\alpha, \beta>0$, then $\mathbb{E}[Y((k-1) \tau, k \tau)]=\exp \left(-(x / \alpha)^{\beta}\right) \alpha^{-\beta}\left[(k \tau)^{\beta}-((k-1) \tau)^{\beta}\right]$. Thus, when the lifetime of a component has a Weibul distribution, the performance of any given PUBM policy can be evaluated exactly in closed form.

\subsection{Optimization}

The optimization of the PUBM policy (minimization of $C^{P U B M}(n, \tau)$ ) is difficult because $C^{P U B M}(n, \tau)$ is usually multi-modal. This is illustrated in Figure 2 for a component with Weibul distributed lifetime. The multi-modal behavior of $C^{P U B M}(n, \tau)$ in $\tau$ for a given $n$ is due to the cost of minimal repairs, as can be seen in Figure 3 for an example with $n=2$. This behavior occurs because the hazard rate of the Weibul distribution grows quickly in time proportional to $x^{\beta-1}$ : If a part is not replaced at the first scheduled down after an initial replacement, then the number of minimal repairs during the second interval of length $\tau$ increases quickly. However, the probability of a component surviving beyond the first scheduled down also decreases to 0 quickly as $\tau$ increases. In this regime, a component will not usually remain functional beyond the first scheduled down so that the high minimal repair costs in the second interval can be avoided.

In general, optimizing a PUBM policy requires enumeration. Usually there are practical reasons to optimize $\tau$ on some grid (e.g. weeks or months). Let $\varepsilon$ denote the granularity of the grid and let $\tau_{m}=m \varepsilon$ for $m \in \mathbb{N}$. A heuristic that is much quicker than enumeration and finds the optimal solution in all cases we have studied is the following: For each $\tau_{m}$ on the grid, one may find a (near) optimal $n_{m}^{*}$ by computing $C^{P U B M}(k, \tau)$ for $k=1, \ldots$ until $C^{P U B M}(k+1, \tau) \geq C^{P U B M}(k, \tau)$ and use $n_{m}^{*}=k$ as the optimal number of downs between planned inspections for the given $\tau_{m}$. This procedure can be further accelerated when the grid size is sufficiently small: if $n_{m}^{*}$ is optimal for $\tau_{m}$, then $n_{m+1}^{*} \in\left\{n_{m}^{*}, n_{m}^{*}-1\right\}$. This procedure (for sufficiently small grid size) is outlined in Algorithm 1.

Finally observe that the best policy parameter setting is $n=1$ and $\tau=\tau^{*}(1)$. However, if $n \neq 1$, then we should choose $\tau$ such that $n \tau \approx \tau^{*}(1)$. This can also be observed in Figure 2 by noting the equivalent property that $\tau^{*}(n) \approx \tau^{*}(1) / n$. 

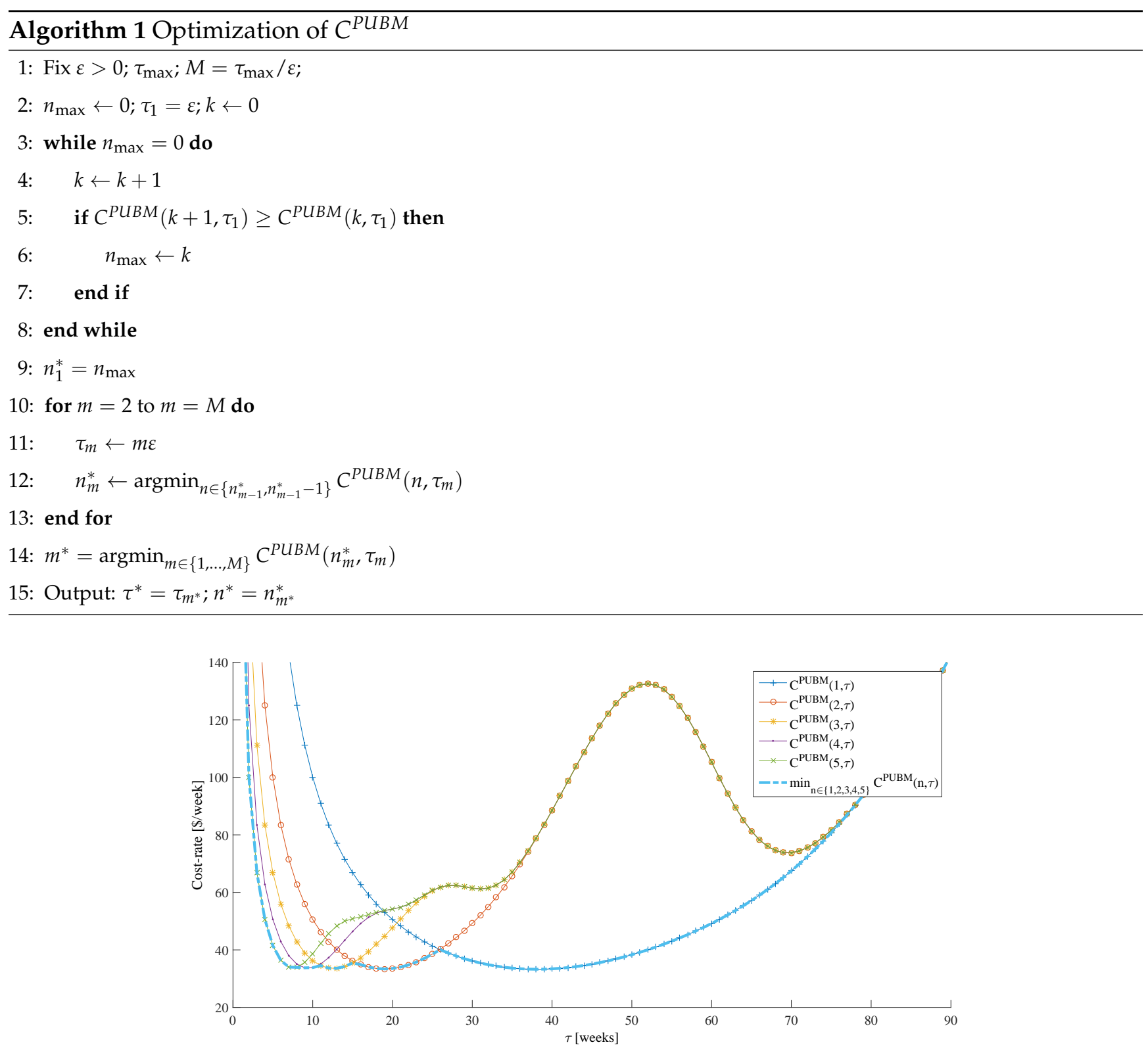

Figure 2: The cost of a PUBM policy for different values of $n$ and optimized over $n \in\{1,2,3,4,5\}$ for a Weibul distribution with shape $\beta=5$ and scale $\alpha=50$ with $C_{p}=1000, C_{u}=1500$, and $C_{r}=600$

\section{Periodic condition based maintenance model}

In this section we study the second single-item policy that we propose. Consider a component that degrades according to a delay time model with exponential time to defect $X$ and delay time $Z$ with corresponding distributions, densities, and failure (hazard) rates: $\mathbb{P}(X \leq x)=F_{X}(x), f_{X}(x)=\frac{d F_{X}(x)}{d x}$, $h_{X}(x)=f_{X}(x) /\left(1-F_{X}(x)\right)$ and analogous definitions for $Z$. Note further that the lifetime of a component under delay time degradation is given by $T=X+Z$. There is a scheduled down every $\tau$ time 


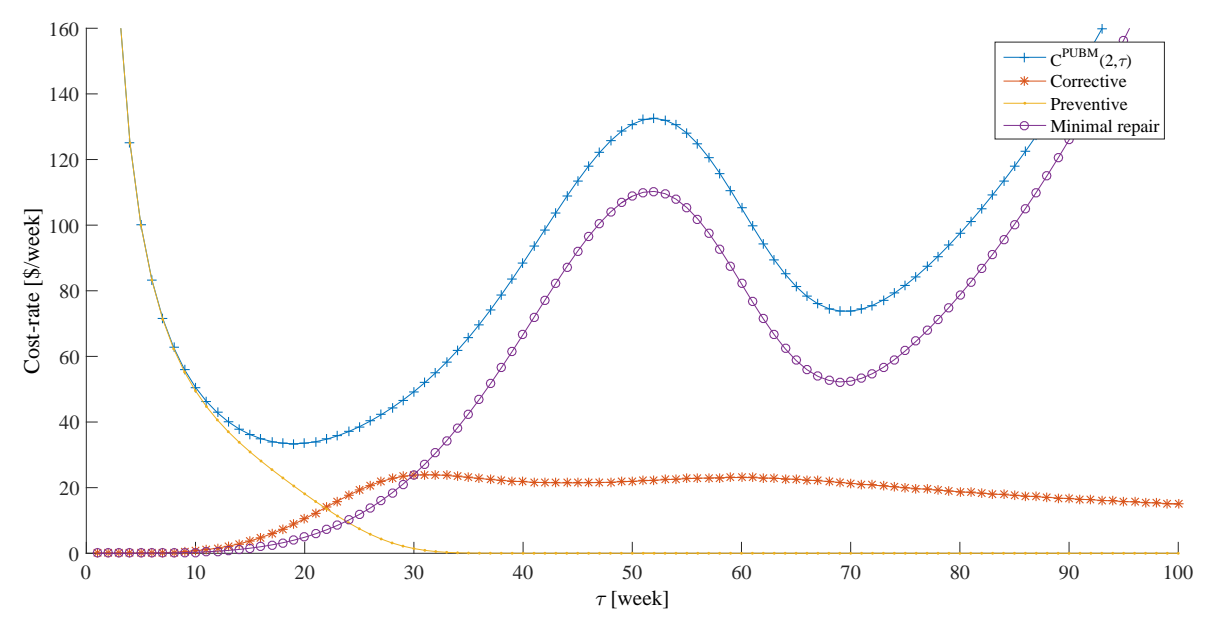

Figure 3: The cost of a PUBM policy for $n=2$ for a Weibul distribution with shape $\beta=5$ and scale $\alpha=50$ with $C_{p}=1000$, $C_{u}=1500$, and $C_{r}=600$. The cost of each term is also shown.

units. During a scheduled down, we can perform an inspection at $\operatorname{cost} C_{i}$. The assumption that $X$ has an exponential distribution fits well in many settings, as explained in Section 1.

We will analyze the following periodic condition based maintenance (PCBM) policy for such components: The component is inspected every $n \tau$ time units, where both $n \in \mathbb{N}$ and $\tau>0$ are policy parameters. If the component is found to be defect during inspection, we apply a preventive replacement at $\operatorname{cost} C_{p} \geq 0$. If the component fails before a defect is found at an inspection, we apply minimal repair at a cost of $C_{r} \geq 0$. This minimal repair will restore the component to a condition that is statistically identical to its state just prior to failure in the sense that its failure rate is restored to the failure rate of the delay time random variable at the time of failure. We thus assume that a minimal repair will never take a component out the defective state. If a minimal repair occurs between scheduled downs, then there is a full replacement at the next scheduled down at the $\operatorname{cost} C_{u} \geq 0$. (Note that an inspection may not have been planned at the next scheduled down.) Usually $C_{u} \geq C_{p}$ because it is more difficult to maintain a component that has been "kept together" by minimal repairs, but our analysis does not require this assumption. Finally, there is an inspection $\operatorname{cost} C_{i} \geq 0$ per inspection epoch. A sample path of this policy is shown in Figure 4. Observe that each time a component is replaced or inspected is a renewal point due to the lack of memory of $X$. Therefore, the PCBM policy can be analyzed with renewal theory.

We note that the PCBM policy may not always outperform other policies for components subject to delay time degradation. However, its periodic nature makes it adept in exploiting economies of scale among different components in the same system. Finally we also note that in practical applications, the delay time degradation model only makes sense if the delay time is short relative to the time to defect. 


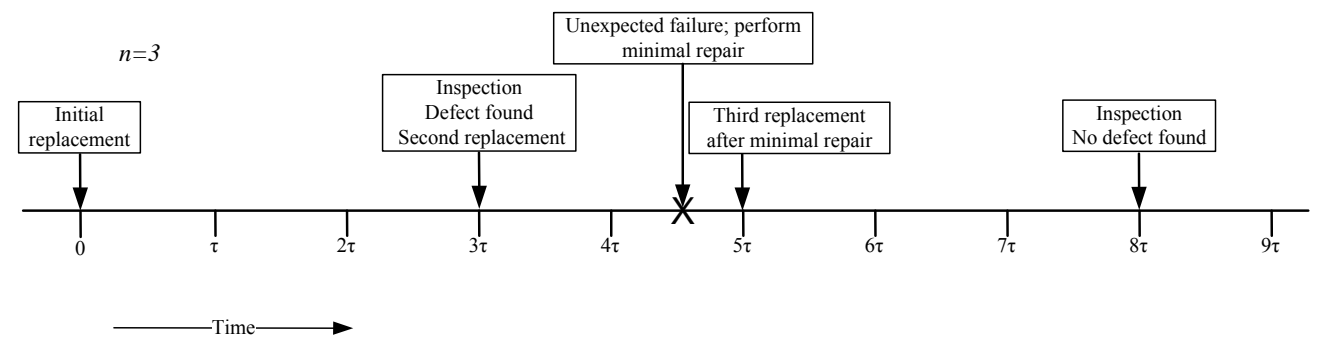

Figure 4: Sample path of a policy with minimal repair between inspection epochs and delay time degradation

\subsection{Performance evaluation}

The expected cycle length under the PCBM policy can be found in the same way as for the PUBM policy and is the same as given in Equation 1:

$$
\begin{aligned}
E C L & =\sum_{k=1}^{n-1} k \tau \mathbb{P}((k-1) \tau \leq T \leq k \tau)+n \tau \mathbb{P}(T \geq(n-1) \tau) \\
& =\sum_{k=1}^{n-1} k \tau\left[F_{T}(k \tau)-F_{T}((k-1) \tau)\right]+n \tau\left(1-F_{T}((n-1) \tau)\right) .
\end{aligned}
$$

Finding the expected costs incurred during a renewal cycle is more involved. The crux is again to determine the expected number of minimal repairs during a renewal cycle. To this end, we define $Y$ as the number of minimal repairs that occur during a renewal cycle.

Proposition 2. The expected number of minimal repairs during a renewal cycle of the PCBM policy satisfies:

$$
\mathbb{E}[Y]=F_{T}(n \tau)+\int_{x=0}^{n \tau} \int_{z=0}^{n \tau-x} \int_{t=z}^{z+\left\lceil\frac{x+z}{\tau}\right\rceil \tau-x} h_{Z}(t) f_{X}(x) f_{Z}(z) d t d z d x,
$$

where $\lceil x\rceil$ denotes $x$ rounded up to the nearest integer.

Proof. Without loss of generality, let us study a renewal cycle starting from time 0 and consider $\mathbb{E}[Y \mid$ $X=x, Z=z]$ rather than $\mathbb{E}[Y]$ directly. Due to the conditioning, the first minimal repair occurs at time $x+z$ if and only if $x+z<n \tau$. Furthermore, if $x+z<n \tau$ then for $t \in(x+z,\lceil(x+z) / \tau\rceil \tau-x \mid)$ minimal repairs occur at rate $h_{Z}(t)$. Alternatively, if $x+z \geq n \tau$, then no minimal repairs occurs during the renewal cycle. Therefore, we have

$$
\mathbb{E}[Y \mid X=x, Z=z]= \begin{cases}1+\int_{t=z}^{\left\lceil\frac{x+z}{\tau}\right\rceil \tau-x} h_{Z}(t) d t, & \text { if } 0 \leq x+z \leq n \tau \\ 0, & \text { otherwise }\end{cases}
$$


Now we can uncondition to find:

$$
\begin{aligned}
\mathbb{E}[Y] & =\int_{x=0}^{\infty} \int_{z=0}^{\infty} \mathbb{E}[Y \mid X=x, Z=z] f_{X}(x) f_{Z}(z) d z d x \\
& =\int_{x=0}^{n \tau} \int_{z=0}^{n \tau-x}\left[1+\int_{t=z}^{\left\lceil\frac{x+z}{\tau}\right\rceil \tau-x} h_{Z}(t) d t\right] f_{X}(x) f_{Z}(z) d z d x \\
& =F_{T}(n \tau)+\int_{x=0}^{n \tau} \int_{z=0}^{n \tau-x} \int_{t=z}^{\left\lceil\frac{x+z}{\tau}\right\rceil \tau-x} h_{Z}(t) f_{X}(x) f_{Z}(z) d t d z d x
\end{aligned}
$$

where the first equality follows from the law of total probability, the second from using equation (6) and using that the length of an inspection interval is $n \tau$, and the third by observing that

$$
\int_{x=0}^{n \tau} \int_{z=0}^{n \tau-x} f_{X}(x) f_{Z}(z) d z d x=\mathbb{P}(T \leq n \tau)
$$

The expected cycle costs can be expressed as:

$$
\begin{aligned}
E C C & =C_{r} \mathbb{E}[Y]+C_{u} \mathbb{P}(T \leq n \tau)+C_{p} \mathbb{P}(X<n \tau \cap X+Y>n \tau)+\mathbb{P}(T \geq n \tau) C_{i} \\
& =C_{r} \mathbb{E}[Y]+C_{u} F_{T}(n \tau)+C_{p} \int_{x=0}^{n \tau}\left(1-F_{Z}(n \tau-x)\right) f_{X}(x) d x+C_{i}\left(1-F_{T}(n \tau)\right) .
\end{aligned}
$$

The expected cost rate of a PCBM policy with parameters $n$ and $\tau$ follows from the renewal reward theorem (Tijms, 2003): $C^{P C B M}(n, \tau)=E C C / E C L$ where ECL is given by equation (5) and ECC is given by equation (8) with $\mathbb{E}[Y]$ as given in Proposition 2.

Remark 2. The case where a component's condition is monitored continuously, but maintenance is only possible at scheduled dows, can be modeled by setting $n=1$ and $C_{i}=0$.

\subsection{Optimization}

The optimization of the PCBM policy (minimization of $\left.C^{P C B M}(n, \tau)\right)$ is difficult. Although $C^{P C B M}(n, \tau)$ appears to be convex in $\tau$ for fixed $n$, clearly $\min _{n \in \mathbb{N}} C^{P C B M}(n, \tau)$ is not, see Figure 5 . However, the same approach used for the minimization of $C^{P U B M}(n, \tau)$ works here too. There are often practical reasons to optimize $\tau$ over a fixed grid (e.g. weeks or months). Let $\varepsilon$ denote the granularity of the grid and let $\tau_{m}=m \varepsilon$ for $m \in \mathbb{N}$. Enumeration is needed for formal optimality guarantees, but the same heuristic that works for the PUBM policy also works here in all cases we studied: For each $\tau_{m}$ on the grid, one may find a (near) optimal $n_{m}^{*}$ by computing $C^{P C B M}(k, \tau)$ for $k=1, \ldots$ until $C^{P C B M}(k+1, \tau) \geq C^{P C B M}(k, \tau)$ and use $n_{m}^{*}=k$ as the optimal number of downs between planned inspections for the given $\tau_{m}$. This procedure can be further accelerated when the grid size is sufficiently small: if $n_{m}^{*}$ is optimal for $\tau_{m}$, then $n_{m+1}^{*} \in\left\{n_{m}^{*}, n_{m}^{*}-1\right\}$. This accelerated algorithm is outlined in Algorithm 2 


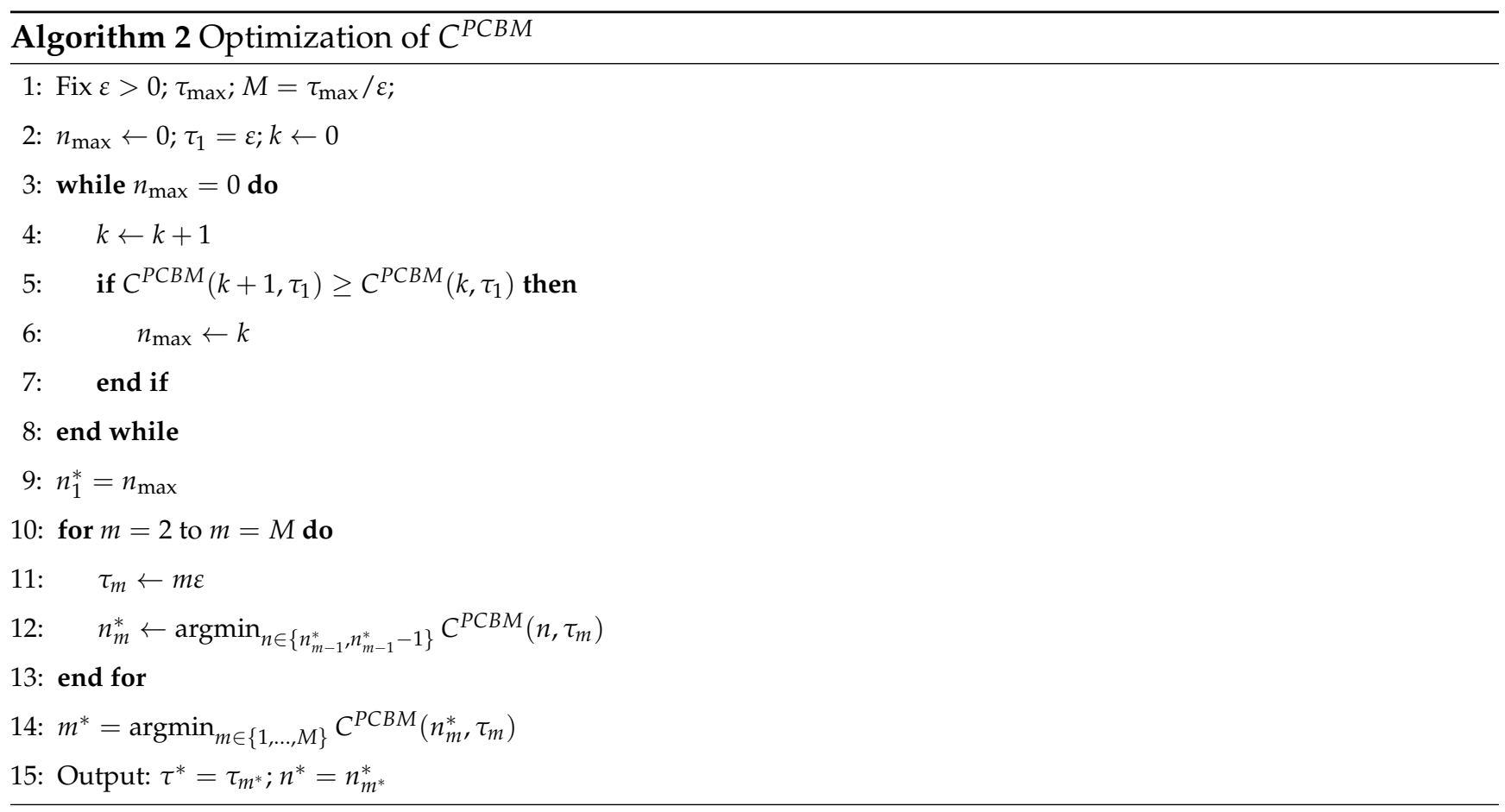

Figure 5 also reveals that $\tau^{*}(n) \approx \tau^{*}(1) / n$ also for the PCBM policy. (Here, $\tau^{*}(n)$ denotes the minimizer of $C^{P C B M}(n, \tau)$ over $\tau$ for a fixed $n$.) The reason for this is analogous to the reason why this holds for the PUBM policy; see Section 3.2.

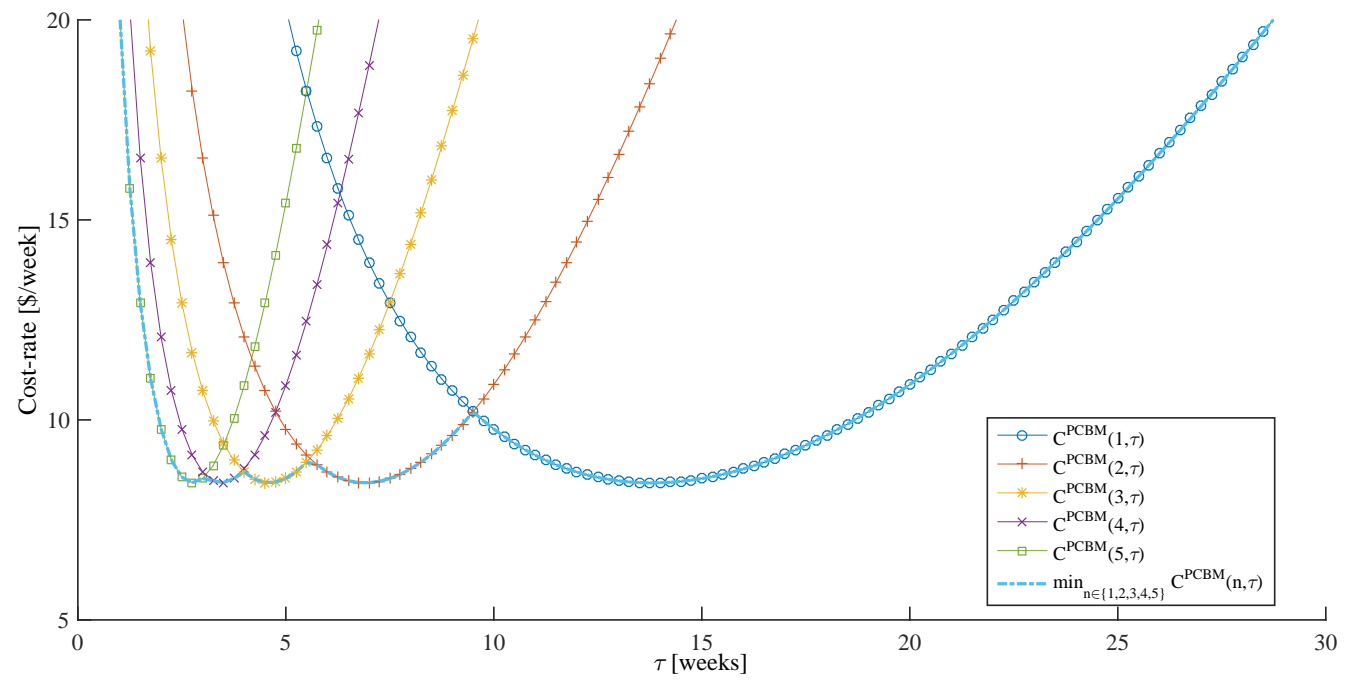

Figure 5: The cost of a PCBM policy for different values of $n$ and optimized over $n \in\{1,2,3,4,5\}$ for an exponential time to defect with mean 15 weeks and a Weibul delay time distribution with shape $\beta=3$ and scale $\alpha=30$ with $C_{p}=1000, C_{u}=1500$, $C_{r}=600$, and $C_{i}=50$. 


\section{Maintenance programs for multi-component system}

In Sections 3 and 4, we have introduced two single-item maintenance models. However, components belong to a system consisting of many components, which means that the maintenance policies per component need to be coordinated. We introduce this optimization problem formally, give a solution approach, and conclude this section with an example.

\subsection{Problem description}

Consider a system consisting of multiple components that are subject to failure. Let $I$ denote the set of all components in a system and let $I_{P C B M}$ denote the set of components for which we apply the PCBM policy and $I_{P U B M}$ denote the set of components for which we apply the PUBM policy; $I_{P C B M} \cap I_{P U B M}=\varnothing$ and $I_{P C B M} \cup I_{P U B M}=I$. The system will have a scheduled down every $\tau$ time units. $\tau$ is a decision variable at the system level. Each scheduled down incurs an expected cost of $C_{d}$ so that the cost-rate due to scheduled downs is $C_{d} / \tau$. Note that this cost term links all components together as they share the scheduled down interval $\tau$.

For each component $i \in I_{P U B M}$, the lifetime $T_{i}$, its distribution $F_{T_{i}}(t)$ and density $f_{T_{i}}(t)$ are given, as well as the cost of a minimal repair $C_{r, i}$, corrective replacement $C_{u, i}$ and planned preventive replacement $C_{p, i}$. We apply the PUBM policy as described in section 3 with $\tau$ as the time between scheduled downs. For each component $i \in I_{P U B M}$, we need to decide on the number of scheduled downs $n_{i}$ before we apply preventive maintenance. We let $C_{i}^{P U B M}\left(n_{i}, \tau\right)$ denote the average cost-rate of a component $i \in$ $I_{P U B M}$ when scheduled downs are $\tau$ time units apart and $n_{i}$ is the number of scheduled downs before component $i$ is replaced preventively. Furthermore, we let $C_{i}^{\text {PUBM* }}(\tau)=C_{i}^{\text {PUBM }}\left(n_{i}^{*}(\tau)\right.$, $\left.\tau\right)$, where $n_{i}^{*}(\tau)$ minimizes $C_{i}^{P U B M}\left(n_{i}, \tau\right)$ over $n_{i} \in \mathbb{N}$ for a given $\tau$.

Components $i \in I_{P C B M}$ are assumed to have an exponential time to defect, $X_{i}$, and generally distributed delay time $Z_{i}$ with corresponding known distributions and densities. Corrective maintenance costs $C_{u, i}$, preventive maintenance costs $C_{p, i}$, and minimal repairs cost $C_{r, i}$ per repair. We control the system using the PUBM policy described in Section 4 . We let $C_{i}^{P C B M}\left(n_{i}, \tau\right)$ denote the expected cost-rate incurred by component $i \in I_{P C B M}$ when scheduled downs are $\tau$ time units apart and inspections are planned $n_{i}$ scheduled downs after replacement. Furthermore, we let $C_{i}^{P C B M *}(\tau)=C_{i}^{P C B M}\left(n_{i}^{*}(\tau), \tau\right)$, where $n_{i}^{*}(\tau)$ minimizes $C_{i}^{P C B M}\left(n_{i}, \tau\right)$ over $n_{i} \in \mathbb{N}$ for a given $\tau$.

The optimization problem that we seek to solve is the following multi-variable non-linear non- 
convex mixed integer programming problem:

$$
\begin{array}{cl}
(P 1) \min _{\tau, n_{i}} & \sum_{i \in I_{P U B M}} C_{i}^{P U B M}\left(n_{i}, \tau\right)+\sum_{i \in I_{P C B M}} C_{i}^{P C B M}\left(n_{i}, \tau\right)+\frac{C_{d}}{\tau} \\
\text { subject to } \quad n_{i} \in \mathbb{N}, \quad \forall i \in I \\
\tau \geq 0,
\end{array}
$$

which is equivalent to the following single variable non-linear non-convex programming problem:

$$
(P 2) \quad \min _{\tau \geq 0} C_{\text {system }}=\sum_{i \in I_{\text {PUBM }}} C_{i}^{\text {PUBM* }}(\tau)+\sum_{i \in I_{P C B M}} C_{i}^{P C B M *}(\tau)+\frac{C_{d}}{\tau}
$$

\subsection{Solution approach}

Optimization problems (P1) and (P2) are non-linear and non-convex, so we cannot hope for a very efficient algorithm. However, for a fixed $\tau$, the objective of (10) can be approximately evaluated by assuming $C_{P U B M, i}\left(n_{i}, \tau\right)$ and $C_{i}^{P C B M}\left(n_{i}, \tau\right)$ are uni-modal in $n_{i}$ for fixed $\tau$ and using a greedy search to find $n_{i}^{*}$ for each $i \in I$. A good solution for optimization problem (P2) can therefore be found by evaluating $C_{\text {system }}(\tau)$ using the scheme above on a sufficiently fine grid $\tau \in\{\varepsilon, 2 \varepsilon, \ldots\}$. In many practical applications, there is a natural grid size, $\varepsilon$, because scheduled downs need to occur at known occasions such as weekends or the end of the month.

\subsection{Example}

Consider a bogie that consists of 6 components with costs and distribution data as indicated in Table 1. The optimal costs for a given $\tau$ for each component is shown in Figure 6. The optimal costs for each component are multi-modal as we also observed in Sections 3.2 and 4.2. The optimal $n_{i}^{*}(\tau)$ for each $\tau$ and component are decreasing in $\tau$ as shown in Figure 7. This is a consequence of our earlier observation that $n_{i}^{*}(\tau-\varepsilon) \in\left\{n_{i}^{*}(\tau), n_{i}^{*}(\tau)-1\right\}$ for sufficiently small $\varepsilon$. The costs for the system as a whole are obtained by summing the costs for each component and adding the cost rate for scheduled downtimes, $C_{d} / \tau$. Figure 8 shows $C_{s y s t e m}$ for the case that $C_{d}=6000$. From this figure, we can see that the optimal time between scheduled downs for the system as a whole is $\tau^{*}=40$ weeks leading to an

optimal system-wide cost of $C_{\text {system }}^{*}=389.04 \$$ /week. The $n_{i}$ for this solution can be read from Figure 7 to be $\left(n_{1}, n_{2}, n_{3}, n_{4}, n_{5}, n_{6}\right)=(1,1,2,1,1,1)$.

Remark 3. The availability is the primary concern of decision makers for some systems. If we let $d_{s}$ denote the (expected) length of a scheduled downtime and $d_{u, i}$ denote the (expected) length of an unscheduled down due the minimal repair of component $i \in I_{P U B M} \cup I_{P C B M}$, then the availability, $A$, of 
Table 1: Input parameters for an example with 6 components

\begin{tabular}{|ll|l|cccc|c|cc}
\hline \multirow{2}{*}{} & & Component & Policy & $C_{p}$ & $C_{u}$ & $C_{r}$ & $C_{i}$ & $\begin{array}{c}\text { Mean time } \\
\text { to defect }\end{array}$ & $\begin{array}{c}\text { Weibul parameters of } \\
\text { life/delay time distribution } \\
\alpha\end{array}$ \\
\hline 1 & bearings & PUBM & 1000 & 1900 & 600 & - & - & 50 & 6 \\
2 & suspension unit & PUBM & 2000 & 3500 & 1300 & - & - & 55 & 4 \\
3 & break unit & PUBM & 3000 & 4500 & 1700 & - & - & 90 & 3.5 \\
4 & front axle & PCBM & 1000 & 1900 & 600 & 50 & 35 & 47 & 4.1 \\
5 & rear axle & PCBM & 500 & 1200 & 450 & 150 & 29 & 40 & 5 \\
6 & compressor & PCBM & 750 & 1100 & 550 & 200 & 40 & 55 & 5 \\
\hline
\end{tabular}
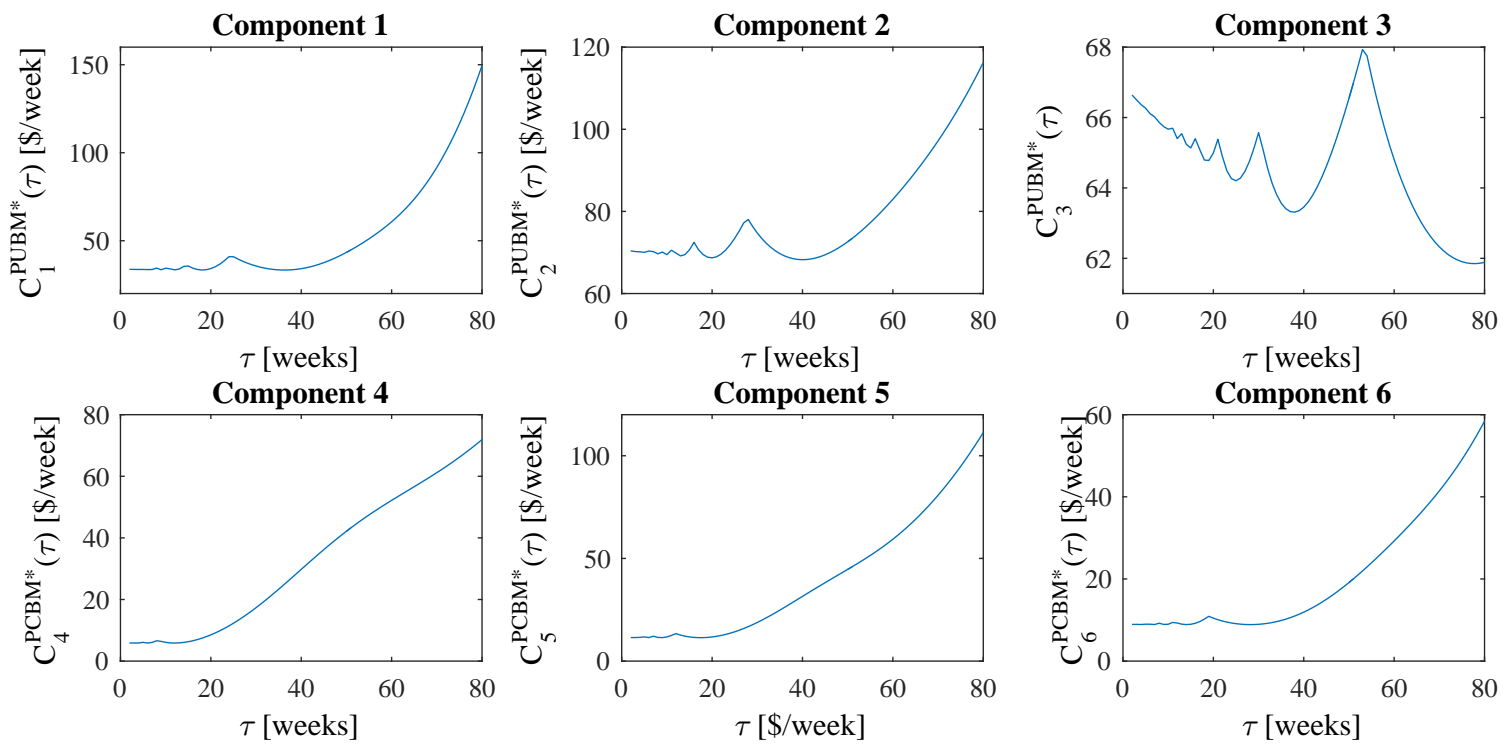

Figure 6: Optimal cost per component for several scheduled down intervals $\tau$.

the system as a whole is given by:

$$
A=1-d_{s} / \tau-\sum_{i \in I_{P U B M}} \frac{\sum_{k=1}^{n_{i}} \mathbb{E}\left[Y_{i}((k-1) \tau, k \tau)\right]}{E C L_{i}} d_{u, i}-\sum_{i \in I_{P C B M}} \frac{\mathbb{E}\left[Y_{i}\right]}{E C L_{i}} d_{u, i}
$$

Decision makers seeking to minimize maintenance cost subject to this availability constraint can use our model and observe that $C_{d}$ and $C_{r, i}$ together serve as Lagrange multipliers of relaxing the availability constraint. Availability targets can then be met efficiently by solving the cost model repeatedly while increasing $C_{d}$ and $C_{r, i}$ until the target is met. 

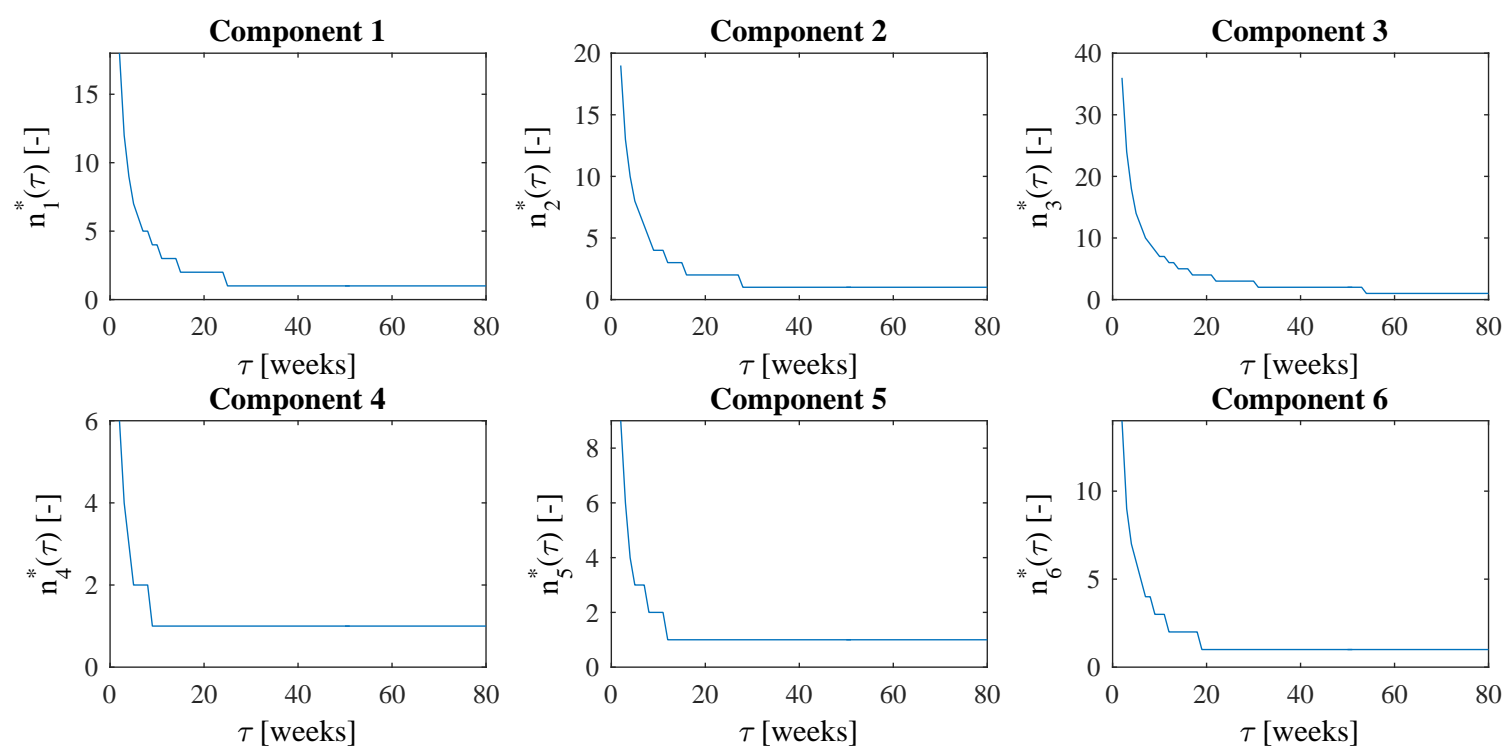

Figure 7: Optimal number of scheduled downs between planned maintenance.

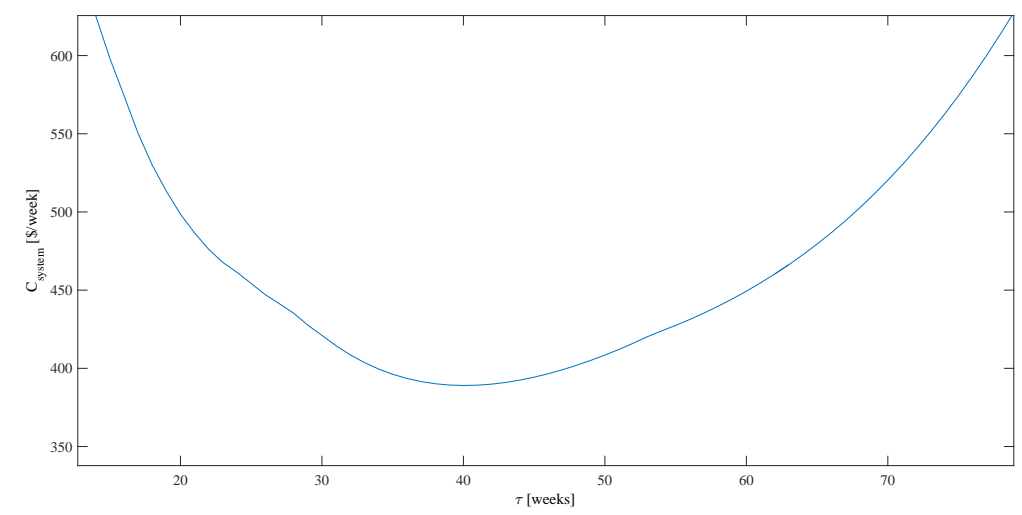

Figure 8: Total optimal cost for the system as a whole for different scheduled down intervals.

\section{Conclusions}

We have proposed two single-item maintenance policies that are convenient to use in a framework for multi-item maintenance optimization. A key assumption that we have made is that there are scheduled downs that can be synchronized over multiple components, during which preventive periodic or condition based maintenance can be performed, while an unscheduled down that results from the failure of one component is used only to minimally repair that one component. This is a realistic assumption if it is very important to have a failed capital asset up as soon as possible or if it is impossible to perform thorough maintenance on the failed component and other components at unscheduled downs. Settings were this assumption holds include maintenance of military equipment during a mission and mainte- 
nance for ships that are sailing. We have explained how the maintenance program for a complete capital asset can be optimized if there are many components using different maintenance policies, and we have given an example.

It would be interesting to combine the models that we have proposed with the models that Zhu (2015) has proposed. He assumes in his models that at an unscheduled down, a complete repair or replacement is performed on the failed component and that other components may be repaired or replaced as well. There are situations where his models fit well and there are situations where our models fit well, but there may also be situations where a minimal repair is interesting for some components, while other components require a complete repair, which gives the opportunity to also repair other components. From a mathematical point of view, we do not expect too many difficulties, except that the total costs cannot be evaluated exactly, since that is not possible with the models of Zhu (2015). Determining all the required inputs for a complete capital asset will be challenging, but the resulting case study should be very interesting.

Combining a maintenance program with other constraints is another interesting possibility for future research. For example, if a user has multiple capital assets, or if the supplier of the capital asset is responsible for the upkeep of many capital assets, then synchronization of the maintenance over the complete installed base becomes relevant. Sometimes clustering will be interesting, for example if systems are geographically close to each other, while spreading out the work is interesting if the work load of the service engineers is taken into account. Rescheduling the exact timing of maintenance at an operational level is also interesting to look at, for instance because of unavailability of service engineers or spare parts.

\section{Acknowledgements}

The first author acknowledges support from the Netherlands Organisation for Scientific Research through a VENI grant.

\section{References}

Bajestani, M. A. and Banjevic, D. (2016). Calendar-based age replacement policy with dependent renewal cycles. IIE Transactions, 48(11):1016-1026.

Barlow, R. and Hunter, L. (1960). Optimum preventive maintenance policies. Operations Research, 8(1):90-100.

Barlow, R. E. and Proschan, F. (1965). Mathematical theory of reliability john wiley \& sons. New York. 
Barron, Y. (2015). Group replacement policies for a rapairable cold standby system with fixed lead times. IIE Transactions, 47(10):1139-1151.

Berg, M. and Epstein, B. (1976). A modified block replacement policy. Naval Research Logistics Quarterly, 23(1):15-24.

Bian, L. and Gebraeel, N. (2014). Stochastic modeling and real-time prognostics for multi-component systems with degradation rate interaction. IIE Transactions, 46(5):470-482.

Castanier, B., Grall, A., and Bérenguer, C. (2005). A condition-based maintenance policy with nonperiodic inspections for a two-unit series system. Reliability Engineering and System Safety, 87(1):109120.

Christer, A. H. (1982). Modelling inspection policies for building maintenance. The Journal of the Operational Research Society, 33(8):723-732.

Christer, A. H. and Waller, W. M. (1984). Delay time models of industrial inspection maintenance problems. The Journal of the Operational Research Society, 35(5):401-406.

de Toledo, M., Freitas, M., Colosima, E., and Gilardoni, G. (2016). Optimal periodic maintenance policy under imperfect repair: A case study on the engines of off-road vehicles. IIE Transactions, 48(8):747758.

Driessen, J., Peng, H., and Van Houtum, G. (2017). Maintenance optimization under non-constant probabilities of imperfect inspections. Reliability Engineering and System Safety, 165:115-123.

He, K., Maillart, L., and Prokopyev, O. (2017). Optimal planning of unpunctual preventive maintenance. IISE Transactions, 49(2):127-143.

Jardine, A. K. S. and Tsang, A. H. C. (2006). Maintenance, Replacement, and Reliability. Theory and Applications. Dekker Mechanical Engineering. CRC Press, Boca Raton (FL).

Jiang, R. (2017). An efficient quasi-periodic inspection scheme for a one component system. IMA Journal of Management Mathematics, 28(3):373-386.

Levi, R., Magnanti, T. L., Muckstadt, J. A., Segev, D., and Zarybnisky, E. J. (2014). Maintenance scheduling for modular systems: Modeling and algorithms. Naval Research Logistics, 61(6):472-488.

Liu, B., Xie, M., and Kuo, W. (2016). Reliability modeling and preventive maintenance of load-sharing systems with degrading components. IIE Transactions, 48(8):699-709. 
Nicolai, R. P. and Dekker, R. (2008). Optimal maintenance of multi-component systems: A review. In Kobbacy, K. A. H. and Murthy, D. N. P., editors, Complex System Maintenance Handbook, Springer Series in Reliability Engineering, pages 263-286. Springer, London (UK).

Olde Keizer, M. C. A., Flapper, S., and Teunter, R. (2017). Condition-based maintenance policies for systems with multiple dependent components: A review. European Journal of Operational Research, in press.

Olde Keizer, M. C. A., Teunter, R. H., and Veldman, J. (2016). Clustering condition-based maintenance for systems with redundancy and economic dependencies. European Journal of Operational Research, 251:531-540.

Pintelon, L. and Van Puyvelde, F. (2006). Maintenance Decision Making. Acco, Leuven (Belgium).

Taghipour, S. and Banjevic, D. (2012). Optimum inspection interval for a system under periodic and opportunistic inspections. IIE Transactions, 44(11):932-948.

Thomas, L. C. (1986). A survey of maintenance and replacement models for maintainability and reliability of multi-item systems. Reliability Engineering, 16(4):297-309.

Tian, Z. and Liao, H. (2011). Condition based maintenance optimization for multi-component systems using proportional hazards model. Reliability Engineering and System Safety, 96(5):581-589.

Tijms, H. (2003). A First Fourse in Stochastic Models. John Wiley \& Sons.

De Jonge, B., Klingenberg, W., Teunter, R. H., and Tinga, T. (2016). Reducing costs by clustering maintenance activities for multiple critical units. Reliability Engineering and System Safety, 145:93-103.

Van Dijkhuizen, G. and Van Harten, A. (1997). Optimal clustering of frequency-constrained maintenance jobs with shared set-ups. European Journal of Operational Research, 99:552-564.

Van Horenbeek, A. and Pintelon, L. (2013). A dynamic predictive maintenance policy for complex multicomponent systems. Reliability Engineering and System Safety, 120:39-50.

Wildeman, R. E., Dekker, R., and Smit, A. C. J. M. (1997). A dynamic policy for grouping maintenance activities. European Journal of Operational Research, 99:530-551.

Yang, L., Ma, X., Peng, R., Zhai, Q., and Zhao, Y. (2017). A preventive maintenance policy based on dependent two-stage deterioration and external shocks. Reliability Engineering and System Safety, 160:201211.

Zhang, N. and Yang, Q. (2015). Optimal maintenance planning for repairable multi-component systems subject to dependent competing risks. IIE Transactions, 47(5):521-532. 
Zhu, Q. (2015). Maintenance optimization for multi-component systems under condition monitoring. PhD thesis, Eindhoven University of Technology.

Zhu, Q., Peng, H., Timmermans, B., and Van Houtum, G. J. (2017). A condition-based maintenance model for a single component in a system with scheduled and unscheduled downs. International Journal of Production Economics, 193:365-380.

Zhu, Q., Peng, H., and van Houtum, G. J. (2016). An age-based maintenance policy using the opportunities of scheduled and unscheduled system downs. BETA Working Paper, 498.

\section{About Joachim Arts}

Joachim Arts is an associate professor at the University of Luxembourg in the Luxembourg Centre for Logistics and Supply Chain Management (LCL) part of the MIT SCALE network. His research interests are in operations research and its applications in supply chain, logistics, maintenance, and business analytics. Much of his research is practice driven as he works with companies such as ASML, Railway operators, Philips, and others. He is particularly interested in research that integrates estimation from data and optimization of decision making. He also studies sustainability issue in multi-tier supply chains.

\section{About Rob Basten}

Rob Basten is an Associate Professor at the Eindhoven University of Technology (TU/e) where he is primarily occupied with maintenance and service logistics and its interfaces. Most of his research focuses on after sales services for capital goods: maintenance policies and maintenance optimization, spare parts inventory control, and design of after sales service supply chains. He is especially interested in using new technologies to improve after sales services. For example, 3D printing of spare parts on location and using improved sensoring and communication technology to perform just in time maintenance. He is further active in behavioral operations management, trying to understand how people can use decision support systems in such a way that they actually improve decisions and add value. Many of Robs research projects are interdisciplinary and in cooperation with (high-tech) industry. 